\title{
Recientes desarrollos en los litigios transnacionales empresas y Derechos Humanos: el caso Kiobel III
}

\author{
Recent Developments on the Transnational Business \\ and Human Rights Litigations: the Kiobel III \\ Maria Chiara Marullo \\ Profesora Ayudante Doctora, Universitat Jaume I \\ marullo@uji.es
}

doi: http://dx.doi.org/10.18543/ced-63-2020pp57-80

Recibido: 6 de abril de 2020

Aceptado: 27 de abril de 2020

Sumario: I. Introducción.-II. Superando la doctrina del foro de necesidad.-III. El caso Kiobel III.-IV. Conclusiones

Resumen: Las víctimas de abusos corporativos encuentran numerosos obstáculos para poder acceder a la justicia, tanto del Estado en cuyo territorio se han verificado los abusos como en el país de origen de las corporaciones. Recientes decisiones de los tribunales europeos, como el holandés en el caso Kiobel III, están abriendo un camino novedoso que, de fructificar, podría suponer un avance muy importante para el futuro de los litigios transnacionales sobre empresas y Derechos Humanos. Previamente a estos casos, los tribunales europeos, aquellos donde las empresas matrices están domiciliadas o tienen su sede principal, abrieron sus puertas en casos de abusos corporativos perpetrados por las filiales en terceros países, basándose en el foro de necesidad o en la prohibición de denegación del acceso a la justicia, al no encontrar otro foro donde las víctimas pudieran hacer valer sus derechos. En los casos citados, los tribunales aceptaron su competencia entendiendo que pueden conocer tanto de los abusos de las empresas matrices, como de las filiales, cuando hay una conexión que permita atraer la filial al estado de origen, apoyando la existencia de un concepto unitario de empresa, donde la matriz toma las decisiones y las estrategias de todas las empresas que pertenecen al grupo y que, por tanto, estarían bajo su control.

Palabras clave: Acceso a la justicia, extraterritorialidad, litigios Transnacionales, empresas y derechos humanos, Kiobel.

Abstract: Victims of corporate abuses encounter numerous obstacles to access justice, both from the State in whose territory the abuses have been verified and in the country of origin of the corporations. Recent decisions of the European Courts, such as the Dutch in the Kiobel III case, are opening a new path that, if successful, could be a very important step forward for the future of transnational litigation on business and human rights. Prior to those cases, the European courts, where the 
parent companies are domiciled or headquartered, opened their doors in cases of corporate abuses perpetrated by subsidiaries in third countries, based on the forum of necessity or the prohibition of access denial to justice, not finding another forum where victims could assert their rights. In the aforementioned cases, the courts accepted their competence understanding that they can know the abuses of matrix and subsidiaries, when there is a connection that allows the subsidiary to be attracted to the state of origin, supporting the existence of a unitary concept of company, where the matrix makes the decisions and strategies of all the companies that belong to the group and that, therefore, would be under their control.

Keywords: Access to justice, extraterritoriality, transnational litigations, business and human rights, Kiobel.

\section{Introducción}

Las empresas multinacionales, (MTNS), protagonistas de primer orden en la esfera internacional, suelen evitar ser responsables de las violaciones de los Derechos Humanos fruto de sus actividades debido, en buena medida, a su insatisfactoria regulación a nivel internacional ${ }^{1}$, a sus complejas estructuras societarias, contractuales y comerciales y a la personalidad jurídica separada de cada empresa que pertenece a la estructura societaria ${ }^{2}$. Pese a ello, están sujetas indudablemente al control de los Estados anfitriones en los que operan y a los que aportan grandes inversiones y, por lo tanto, la vía legal más obvia a través de la cual buscar reparación en casos de abusos de Derechos Humanos relacionados con ellas es recurriendo al forum delicti commiss $i^{3}$. Sin embargo, suele suceder o bien que las empresas locales no tengan los medios para poder hacer frente a las reparaciones

1 Vid.: Carmen Márquez Carrasco, «Las relaciones entre el Derecho internacional y la práctica interna en el ámbito de los derechos humanos y la responsabilidad de las empresas», Anuario Español de Derecho Internacional 34, (2018): 707-728. Sobre el tema del Borrador de Tratado de Empresas y Derechos Humanos, vid., Antoni Pigrau Solé y Daniel Iglesias Márquez, «La versió revisada de l'Esborrany del Tractat sobre Empreses i Drets Humans: cap a la següent ronda de negociacions», Policy Paper ICIP , n. ${ }^{\circ} 19$ (2019).

2 Vid. Álvarez Rubio, Juan José y Katerina Yiannibas, (editors), Human Rights in Business: Removal of Barriers to Access to justice in the European Union, (Londres: Routledge, 2017).

${ }^{3}$ Regla especial de competencia judicial internacional en materia extracontractual «tribunal del lugar donde se hubiere producido o puede producirse el hecho dañoso» Sobre este tema: Laura Carballo Piñeiro, «Litigación internacional y daños al ambiente», Revista Ítalo-española de Derecho Procesal 1, (2018), http://www.rivitsproc.eu/wp-content/ uploads/2018/10/laura_carballo.pdf. Acceso el 10 de diciembre de 2019. 
de los daños derivados por sus actividades o bien que los Estados anfitriones carezcan de la capacidad o la voluntad de cumplir con su obligación de garantizar que las víctimas de abusos corporativos tengan acceso a la justicia, favoreciendo con ello la impunidad ${ }^{4}$.

En ocasiones, esta situación ha dado lugar a litigios paralelos ante diversos tribunales. La tendencia ha sido recurrir a los sistemas legales de los Estados de origen de las MTNS ${ }^{5}$, donde la empresa matriz tiene su sede estatutaria, administrativa o principal actividad ${ }^{6}$, para conocer de violaciones cometidas extraterritorialmente por empresas locales que pertenezcan a la misma estructura empresarial ${ }^{7}$. Así, por ejemplo, en el ámbito europeo los tribunales de origen han reconocido su competencia principalmente sobre la base del llamado foro de necesidad, abriéndose a las demandas al no encontrarse otro foro disponible, con base en la prohibición de denegación de justicia o del derecho a un juicio justo en virtud del artículo 6 (1) del $\mathrm{CEDH}^{8}$.

${ }^{4}$ Vid. Maria Chiara Marullo y Francisco Javier Zamora Cabot, Derechos Humanos y empresas: temas actuales. (Napoli: SCIENTIFICA, 2018).

5 Vid. Juan José Álvarez Rubio y Katerina Yiannibas, «Access to justice for businessrelated Human Rights», en La Unión Europea y la protección de los derechos fundamentales ed. por Francisco De Asís Peña Díaz, Ana Salinas de Frías, (dir coord. por Alejandro Frías Sánchez), Enrique J Martínez Pérez,. (dir.), (Valencia: Tirant lo Blanch,2018), 91-110 y Daniel Iglesias Marquez y Maria Chiara Marullo, «Seeking Access to Justice for Victims of Corporate Abuses through the Sustainable Development Goals», en Rethinking Sustainable Development in Terms of Justice: Issues of Law, Theory and Governance. (Cambridge: Cambridge Scholars Publishing, 2018), 120-145.

${ }^{6}$ Bajo el artículo 63 del Reglamento Bruselas Bis, los tribunales de un Estado Miembro podrán conocer sobre demandas contras empresas que estén domiciliadas en la UE: «A efectos del presente Reglamento, se entenderá que una sociedad u otra persona jurídica está domiciliada en el lugar en que se encuentra: a) su sede estatutaria; b) su administración central o, c) su centro de actividad principal». https://www.boe.es/doue/2012/351/L0000100032.pdf. Acceso el 9 de diciembre de 2019.

7 Vid. Daniel Augenstein y David Kinley, «When Human Rights 'Responsibilities' become 'Duties': The Extra-Territorial Obligations of States that Bind Corporations», en Human Rights obligations of business: beyond the corporate responsibility to respect?, ed. por David Bilchitz, \& Surya Deva (Cambridge: Cambridge University Press, 2013).

${ }^{8}$ En este sentido véase Arnaud Nuyts, Study on Residual Jurisdiction (Review of the Member States' Rules concerning the "Residual Jurisdiction» of their courts in Civil and Commercial Matters pursuant to the Brussels I and II Regulations), General Report (Final Version 3 September 2007)63, disponible para consulta en https://www.econbiz. de/Record/study-residual-jurisdiction-review-member-states-rules-concerning-residualjurisdiction-courts-civil-commercial-matters-pursuant-brussels-regulations/10010527847 y art. 3 II. Emergency jurisdiction Switzerland's Federal Code on Private International Law (CPIL) of December 18, 1987. Vid. asimismo, Report: Comparative Study of «Residual Jurisdiction» in civil and commercial disputes in the EU National Report for: France. Section 16, p. 19. Véase también Gwynne Skinner. Robert McCorquodale, Olivier De Schutter, The Third Pillar: Access to Judicial Remedies for Human Rights Violations by Transnational Business, 2013, accessible en https://static1.squarespace. 
En los últimos años en varios tribunales europeos hay un cambio de posicionamiento al entender las estructuras societarias desde un enfoque unificado $^{9}$, abriendo las puertas a las víctimas de graves violaciones de los Derechos Humanos cometidas extraterritorialmente, es decir, fuera de la UE. En otras palabras, estiman que su jurisdicción no se fundamenta únicamente en un estado de indefensión de las víctimas, sino también por la existencia de un nexo entre la conducta delictiva y la empresa matriz, en algunos casos con fundamentación en el Reglamento Bruselas bis arts. 4 (1) y 63 (1), respecto de ésta y, de forma residual, tratándose de las filiales, en el derecho interno de los Estados Miembros, en la medida en que permita atraerlas a la competencia de los foros de origen ${ }^{10}$.

Las tendencias de esos tribunales demuestran que el nexo aludido, requisito para entablar las demandas, podría darse por cumplido demostrando un vínculo entre la empresa madre y la filial o sucursal, que estarían bajo su control o influencia. Por lo que las víctimas tienden a demandar a las empresas matrices, bien por complicidad en los abusos o bien por no haber cumplido con su deber de vigilancia y evitar impactos de las actividades de sus cadenas de suministro, y accionar también frente a las filiales locales, que son las que finalmente se ven involucradas con mayor intensidad en los

com/static/583f3fca725e25fcd45aa446/t/58657dfa6a4963597fed598b/148304639204/ The-Third-Pillar-FINAL1.pdf, Acceso el 10 de diciembre de 2019. Nicola Jägers, Katinka Jesse, y Jonathan Verschuuren, «The Future of Corporate Liability for Extraterritorial Human Rights Abuses: The Dutch Case Against Shell», American Journal of International Law (2014), 39-40. Entre los casos más relevantes mencionamos: Abood/Kuwait Airways Corp., Amsterdam Sub-District Court (5 January 1996), Nederlands Internationaal Privaatrecht 145, 222 (Kuwait Airways case I); Lubbe v. Cape Plc [2000] 1 WLR 1545, Accueil Procès COMILOG (Gabon), 10 September 2015, the Paris Court of Appeal.

${ }^{9}$ El cambio se puede ver también desde la perspectiva regulatoria, véanse por ejemplo los nuevos desarrollos en el tema de la fiscalidad internacional y la propuesta de la OCDE, por ahora relativa a las empresas digitales y que podría suponer un cambio radical en el sistema fiscal internacional, un modelo unificado para gravar a empresas multinacionales independientemente de su sede fiscal, administrativa o estatutaria. El grupo de trabajo constituido en marzo de 2017 entiende que los impactos sobre las comunidades y los beneficios de estas empresas se refieren a todo el grupo empresarial, por lo que los Estados podrían exigir el pago de impuestos a una compañía desde el momento que tenga una determinada cantidad de ingresos en su territorio. Una redistribución de la riqueza para establecer unas reglas fiscales internacionales más respetuosas de la competencia y al mismo tiempo más justas en términos de desarrollo sostenible de las comunidades OCDE para los gigantes digitales; vid., más información en: «La OCDE propone que los gigantes digitales paguen impuestos donde estén sus usuarios», RTVE, Acceso el 11 de noviembre de 2019, http://www.rtve.es/noticias/20191009/ocde-propone-gigantes-digitales-paguen-impuestosdonde-esten-usuarios/1981147.shtml.

10 Vid. Christine Salamanca Mandap, Jurisdiction of Parent Companies' Home State Courts Over Foreign Subsidiaries Abroad: A Comparative Approach Between The Netherlands and The United Kingdom, vol. 11, n. ${ }^{\circ} 2$ (Amsterdam Law Forum,2019). 
hechos. Cabe mencionar que en muchos casos el recurso a estos tribunales del Estado de origen puede ser la única opción disponible para entablar acciones indemnizatorias o de reparación de los daños causados.

Estas páginas se centran principalmente en los litigios civiles ante tribunales europeos ${ }^{11}$, en particular los casos Kiobel III ${ }^{12}$, por los destrozos medioambientales y otras violaciones de derechos humanos perpetradas en Nigeria. En este país hay dos grandes compañías petroleras, la Anglo-Dutch Company, Royal Dutch Shell (Shell) y Eni Oil de Italia, acusadas desde hace décadas de daños medioambientales. Una situación que ha originado numerosos conflictos entre las comunidades locales, el Gobierno de Nigeria y las empresas locales y matrices y, al mismo tiempo, a entablar demandas en Nigeria y fuera de este país. En tales juicios se pretenden esclarecer principalmente tres cosas: qué rol ha tenido el gobierno de Nigeria o qué medidas ha activado para reducir los impactos de las actividades de extracción de petróleo en las comunidades locales, y cuáles han sido las medidas activadas para reducir los efectos de la contaminación y garantizar los medios de subsistencia a sus habitantes. Y, por otra parte, evaluar cómo han sido gestionados los problemas medioambientales a través de las empresas locales y las matrices, para determinar si hay responsabilidad directa de ambos tipos de empresas.

Estos casos están suscitando un gran interés, en términos de acceso a la justicia y por sus múltiples implicaciones, entre la que destaca el importante tema del reconocimiento de los documentos relativos a los hechos probados ante un tribunal extranjero, como sucede en el caso Kiobel III y, en la especie, los tribunales de los Estados Unidos en Kiobel I y II. Sin embargo, debe tenerse en cuenta que el marco legal que gobierna este tipo de litigios es altamente complejo, ya que abarca diferentes conjuntos de normas legales nacionales e internacionales; cada Estado tiene su propio sistema legal y a nivel europeo no se ha logrado la armonización esperada en estas materias. La efectividad del recurso a estos tribunales dependerá de muchos factores, como la fortaleza institucional de los Estados de la empresa matriz y la capacidad y la independencia de los diferentes sistemas judiciales.

11 Vid. Jonas Grimheden, «Civil Litigation in response to corporate human rights abuses: the European Union and its Member States», Case Western Reserve Journal of International Law,Vol.50, Issue I, (2018). Burkhard Hess y Martina Mantovani , «Current developments in forum access: Comments on jurisdiction and forum non conveniens», MPIL Research Paper Series, N. ${ }^{\circ} 2019$ (1).

12 Vid. Maria Chiara Marullo, y Francisco Javier Zamora Cabot, «Transnational human rights litigations. Kiobel's Touch and Concern: a test under construction», Papeles el Tiempo de los Derechos, n. $^{\circ} 1$ (2016). 


\section{Superando la doctrina del foro de necesidad}

Frente a la imposibilidad de garantizar el acceso a la justicia, entendido como acceso a los tribunales en los países en los que se verifican las actividades delictivas, y a la imposibilidad de presentar los casos ante tribunales internacionales ${ }^{13}$, es notoria la práctica de algunos tribunales europeos o canadienses, entre otros, de conocer casos fundamentando, como hemos dicho, su jurisdicción en la doctrina del foro de necesidad o forum necessitatis, siempre que concurran otros requisitos entre los que destaca el nexo o vínculo entre los hechos y el Estado del foro. A título de ejemplo, la doctrina del forum necessitatis proporcionó una base para que el Tribunal de Distrito de La Haya se declarase competente para entender de una demanda civil planteada por un demandante extranjero por haber sido encarcelado ilegalmente y torturado en Libia. En este caso, la única conexión que existía

${ }^{13}$ En otras ocasiones hemos expresado nuestra opinión en relación con la necesidad de modificar el Estatuto del Tribunal Penal Internacional para que este último pueda conocer casos que involucren la responsabilidad de entes privados. En septiembre de 2016, en el documento relativo a las priorizaciones del Tribunal, los fiscales afirmaron que iban a dar una especial consideración a la comisión de crímenes de lesa humanidad y de genocidio, cuando se realizaran o produjeran el resultado de acaparamiento de tierras y de recursos naturales, a través de empresas transnacionales. Más información sobre el documento: «Policy paper on case selection and prioritisation», International Criminal Cort, Acceso el 09 de noviembre de 2019, https://www.icc-cpi.int/itemsDocuments/20160915_OTP-Policy_Case-Selection_Eng. pdf. Cabe mencionar que en la propuesta inicial para el establecimiento de un Tribunal Penal Internacional y la creación de un Tratado Internacional para su constitución, el artículo 23, en su versión previa a la actual establecía: «5. La Corte también ejercerá jurisdicción sobre las personas jurídicas, a excepción de los Estados, cuando los crímenes se hayan cometido en nombre de ellas o por sus agentes o representantes. 6. La responsabilidad penal de las personas jurídicas no excluirá la responsabilidad penal de las personas naturales que hayan actuado en calidad de autores o cómplices de esos mismos crímenes». Sobre este artículo, en la nota pie 3 (p.49) se manifiesta que: «Existe una profunda divergencia de opiniones por lo que se refiere a la conveniencia de incluir en el Estatuto la responsabilidad penal de las personas jurídicas. Muchas delegaciones se oponen firmemente a ello, en tanto que algunas son firmemente partidarias de la inclusión. Otras mantienen una posición abierta. Algunas delegaciones consideraron que el hecho de incluir únicamente la responsabilidad civil o administrativa de las personas jurídicas podría constituir una solución intermedia. No obstante, esa posición no se ha examinado detenidamente. Varias delegaciones partidarias de la inclusión de las personas jurídicas consideraron que el término en cuestión debería hacerse extensivo a las organizaciones sin personalidad jurídica». Vid., más información relativa al Informe del Comité Preparatorio sobre el establecimiento de una Corte Penal Internacional, A/CONF.183/2/ Add.1, 14 de abril de 1998, accesible en https://www.dipublico.org/conferencias/cortepenal/ A-CONF.183-2-Add.1.pdf. Acceso el 11 de noviembre de 2019. No obstante, no hay duda que haya corrientes nuevas, ya que las últimas decisiones del Tribunal han empezado a evaluar la capacidad de las empresas en la comisión de graves delitos, utilizando el concepto de organización empleado en el artículo 7 del Estatuto de Roma, relativo al crimen de lesa humanidad y, en particular, en el supuesto de ataque contra la población civil. 
con los Países Bajos era la presencia del demandante en el país ${ }^{14}$. Recientemente, siempre en Holanda se ha presentado una demanda basada sobre el foro de necesidad contra el Chief of the General Staff Benjamin Gantz:

The claim invokes the Dutch forum necessitatis rule (Article 9 CPR; other European States have similar rules), often also known as 'universal jurisdiction' however clearly the rule has its constraints. Claimant's lawyer, Meester Liesbeth Zegveld, argues the application of the rule here. The piece includes assessment of sovereign immunity, and the involvement of Article 6 ECHR. Its outcome will also play a role in issues of corporate social responsibility and jurisdiction ${ }^{15}$.

Por su parte, los tribunales franceses han tratado el tema, entendiendo que Francia era el último recurso para las víctimas ${ }^{16}$. En 2010, la Fundación

${ }^{14}$ Demanda por daños y perjuicios presentada por un médico palestino que alegaba haber sufrido daños después de permanecer encarcelado ilegalmente durante ocho años en Libia acusado de haber infectado a niños con VIH/SIDA. Rechtbank's-Gravenhage [Tribunal de distrito de La Haya], 21 de marzo de 2012, LJN: BV9748, El-Hojouj/Unnamed Libyan Officials, Países Bajos. El artículo 9 del Código de procedimiento civil establece: Article 9 Tacit choice of forum ('forum necessitatis') When Articles 2 up to and including 8 indicate that Dutch courts have no jurisdiction, then they nevertheless have if: a. the case concerns a legal relationship that only affects the interests of the involved parties themselves and the defendant or a party with an interest in the legal proceedings has appeared in court, not exclusively or with the intention to dispute the jurisdiction of the Dutch court, unless there is no reasonable interest to conclude that the Dutch court has jurisdiction. b. a civil case outside the Netherlands appears to be impossible, or; c. the legal proceedings, which are to be initiated by a writ of summons, have sufficient connection with the Dutch legal sphere and it would be unacceptable to demand from the plaintiff that he submits the case to a judgment of a foreign court. Más información en: http://www.dutchcivillaw.com/legislation/ civilprocedure001.htm. Acceso el 07 de noviembre de 2019.

15 Más información en Geert Van Calster,14 de Enero de 2020, «Suing the Chief of the Israeli general staff in the Netherlands.Ismail Ziada v Benjamin Gantz tests Dutch forum necessitates rules», Gavc Law-Geert Van Calster, acceso el 15 de enero 2020, https:// gavclaw.com/2020/01/14/suing-the-chief-of-the-israeli-general-staff-in-the-netherlandsismail-ziada-v-benjamin-gantz-tests-dutch-forum-necessitatis-rules/.

16 Cass. Civ. 1ère, 19 November 1985, Cognacs and Brandies, JDI 1986. «Denial of justice is, indeed, an admissible ground of jurisdiction for French courts. Yet, in order for the system to remain balanced and for France not to become the «last resort» in any case, whatever the circumstances, case law has provided for two conditions to be met: - the plaintiff relying on French courts' jurisdiction has to prove that it is impossible for him or her to bring his or her claim before a foreign court. Impossibility may be based on either factual (e.g. plaintiff facing major threats if putting foot on foreign soil) or legal grounds (e.g. the foreign court has already found it has no jurisdiction). French courts will also admit impossibility to seize a foreign court where, upon examination of foreign rules on jurisdiction of the states concerned, they find that no foreign court will retain its jurisdiction $[\ldots] ;-$ the dispute has to bear some link with France. How important this link has to be remains debated: whereas case law seems to be satisfied with the plaintiff's having a stable residence in 
Interamericana de Abogados reconoció expresamente la importancia de la creación de foros de necesidad para garantizar el acceso a la justicia y propuso su inclusión en un proyecto latinoamericano: la Ley Modelo sobre la Protección Internacional de los Derechos Humanos (La Ley Modelo Dahl) cuyo Articulo 1 contenía una disposición de foro de necesidad ${ }^{17}$.

Pero cabe indicar, sin embargo, que esta doctrina ha encontrado fuertes detractores, entre los que destaca el propio Tribunal Europeo de Derechos Humanos. Recordamos al respecto que en el reciente caso Naït-Liman este Alto Tribunal ha establecido que Suiza no estaría obligada a conocer demandas basadas en el forum necessitatis o en el principio de jurisdicción universal en materia civil, ya que no existe un consensus generalizado en el Derecho Internacional sobre esta obligación incluso tratándose, como sucedía en el caso referenciado, de graves violaciones de normas de jus cogens ${ }^{18}$.

Efectivamente, en la actualidad no existe una norma internacional unívoca que establezca la obligatoriedad o la facultad de los Estados para la activación de dicho mecanismo. Además, no se ha registrado praxis estatal consistente y orientada a crear un modelo único y obligatorio de foro de necesidad. Lo que ha dado lugar a decisiones diferentes y en algunas ocasiones, contradictorias. De hecho, dentro de la misma Unión Europea, la norma que establece en materia civil y mercantil la competencia de los Tribunales de los Estados parte de la Unión, el Reglamento Bruselas Bis ${ }^{19}$, relativo a la

France, some authors are in favor of retaining jurisdiction even if said link is more remote (e.g. presence of financial or other interests in France, obligation to be performed in France)». Vid., más información en:

Comparative study of «residual jurisdiction» in civil and commercial disputes in the EU National Report for: France. El informe se puede consultar en: https://ec.europa.eu/civiljustice/news/docs/study_resid_jurisd_france_en.pdf. Acceso el 07 de noviembre de 2019.

17 Sobre este tema véanse: Juan José Álvarez Rubio et all, «A Propósito de la Ley Modelo Latinoamericana de Protección Internacional de los Derechos Humanos (La Ley Modelo Dahl)», Revista de Estudios Jurídicos, n. ${ }^{\circ} .11$ (2011): 1-15.

18 Tribunal Europeo de Derechos Humanos, caso n. 51357/07, Naït-Liman c. Svizzera, en: http://hudoc.echr.coe.int/. Acceso el 08 de noviembre de 2019. Sobre este tema, vid. Edoardo Benvenuti, «Quale tutela del diritto di accesso alla giustizia civile per le vittime di gravi violazioni dei diritti umani? Riflessioni a margine della decisione della Grande Camera della Corte Europea nel caso Nait-Liman», Ordine internazionale e diritti umani, (2018): 322-332. También, Daniel Rietiker, «The Case of Naït-Liman v. Switzerland Before the European Court of Human Rights: Where Are the Limits of the Global Fight Against Torture?», n.d. Acceso el 07 de noviembre de 2019, https://harvardilj.org/2019/03/the-caseof-nait-liman-v-switzerland-before-the-european-court-of-human-rights-where-are-the-limitsof-the-global-fight-against-torture/\#_ftn 1 .

19 Reglamento (UE) no 1215/2012 del Parlamento Europeo y del Consejo de 12 de diciembre de 2012 relativo a la competencia judicial, el reconocimiento y la ejecución de resoluciones judiciales en materia civil y mercantil (refundición). El texto se puede consultar en: https://www.boe.es/doue/2012/351/L00001-00032.pdf. Acceso el 11 de noviembre de 2019. 
competencia judicial, el reconocimiento y la ejecución de resoluciones judiciales en materia civil y mercantil, no incluye una cláusula para conocer casos extraterritoriales fundamentando la competencia en la doctrina del foro de necesidad. Así, durante el proceso de modificación del Reglamento de Bruselas, los Estados tuvieron la oportunidad muy clara de impulsar la creación de una norma sobre tal foro, en los casos en los que otros resultasen inviables para las víctimas de graves ilícitos internacionales perpetrados por empresas multinacionales fuera de la Unión, pudiéndose haber modificado el artículo 26 ante una propuesta de cláusula específica sobre forum necessitatis que, no obstante, careció del apoyo necesario ${ }^{20}$. En sus términos, tal propuesta hubiera permitido al órgano jurisdiccional nacional de un Estado Miembro hacer valer su competencia en el caso de que ningún otro tribunal se la hubiera atribuido, estableciendo un vínculo mínimo con el foro.

Como dijimos, para superar los límites de la doctrina del foro de necesidad y encontrar otros mecanismos con el fin de garantizar el acceso a la justicia, en los últimos años varios tribunales europeos han abierto las puertas a las víctimas extranjeras de violaciones cometidas extraterritorialmente, cuando exista un nexo entre la conducta delictiva y la empresa matriz, en algunos casos con fundamentación en el Reglamento de Bruselas bis art. 4 (1) 63 (1) y, en otros, de forma residual en el derecho interno, en la medida en que permita atraer a las filiales a la competencia de los foros de origen de la matriz, siendo paradigmático el caso Vedanta ${ }^{21}$ respecto de las sedes judiciales inglesas, por sus novedosos planteamientos y las repercusiones que están teniendo en todos los ámbitos. Cabe destacar que los tribunales ingleses han reinterpretado previamente el concepto de corporate liability:

[the] control of a business partner's activity by the EU-based multinational corporation [...] can be de facto or, maybe, presumed based on a duty to control. The core concept of control is supplemented

20 Vid. Lucas Roorda and Cedric Ryngaert, «Business and Human Rights Litigation in Europe and Canada: the Promises of Forum Necessity Jurisdiction», Rabels Journal of Comparative and International Private Law 80, (2018):783-804.

${ }^{21}$ Lungowe and Ors. v Vedanta Resources Plc and Konkola Copper Mines Plc [2017] EWCA Civ 1528. En este caso se demanda a la empresa minera con sede en el Reino Unido, y su filial zambiana Konkola Copper Mines (KCM) por contaminación que ha, entre otras cosas causado enfermedades y dañado los cultivos. Este tribunal ha afirmado que sería prácticamente imposible para los afectados obtener justicia en Zambia, uno de los países más pobres del mundo. Además, el sistema de justicia en este país no tiene los instrumentos legales para poder manejar un caso tan difícil contra empresas multinacionales y sus filiales. Más información en: Russel Hopkins, 26 de Julio de 2019 «Bringing responsibility home: what next for parent company liability? Bright Line Law», Lexology, https://www.lexology. com/library/detail.aspx?g=e9a836c4-d521-49df-b043-e074e7279cc7. Acceso el 30 de octubre de 2019. 
by the familiar concept of knowledge, actual or presumed - knowledge of risks may trigger a duty to intervene» ${ }^{22}$.

La sentencia contiene varios aspectos relevantes, sobre todo bajo la óptica de futuros casos relativos a la forma en que las multinacionales gestionan sus riesgos relacionados con el medioambiente y los Derechos Humanos en sus cadenas de suministro y, seguramente estaríamos frente a un dilema para las empresas, porque la publicación de informes no financieros podría tener importantes implicaciones en términos de responsabilidad corporativa del grupo:

Concerning duty of care, the Vedanta judgment is undoubtedly a critical development in holding parent companies to account for their corporate social responsibility-type statements. Some commentators have argued that the findings in Vedanta of the significance of corporate policies for parent company duty of care and potential liability may have a 'chilling effect' on companies' willingness to set and implement human rights and environmental policies centrally. The Vedanta judgment appears to catch parent companies on the horns of a dilemma. Investors, civil society organizations and, in some cases, regulators expect companies to publish information regarding their management of their subsidiaries $^{23}$.

Así, opinamos que este caso está llamado a constituir un precedente de relevancia mundial, dado que por primera vez un sistema judicial con tanto prestigio afronta el tema de la responsabilidad directa hacia los perjudicados de las empresas matrices por la actividad de sus filiales en el extranjero, en materia medioambiental, basándose en el derecho ordinario de ilícitos civiles, aquí el common law of torts ${ }^{24}$.

22 Penelope A. Bergkamp, «Models of Corporate Supply Chain Liability», (Master Thesis, KU Leuven,2018-2019) https://www.scriptiebank.be/sites/default/files/thesis/201910/ Scriptie \%20Penelope \%20A.\%20Bergkamp\%20-\%20Models\%20of\%20Corporate \%20 Supply\%20Chain\%20Liability\%202019.pdf. Acceso el 30 de octubre de 2019.

${ }_{23}$ Marilyn Croser, Martyn Day, Mariëtte Van Huijstee y Channa Samkalden, «Developments in the Field, Vedanta v Lungowe and Kiobel v Shell: The Implications for Parent Company Accountability», Business and Human Rights Journal Cambridge University Press, (2019): 1-7. La altura de los tiempos y la marcada tendencia hacia la responsabilización de las empresas a partir de criterios de transparencia en la gestión de sus actividades desaconsejarían, sin embargo, una gestión empresarial contraria a estos desarrollos, si solo fuera por la más que probable pérdida de imagen que conllevaría.

${ }^{24}$ Cabe recordar que la práctica de las sedes federales de los Estados Unidos en base al Alien Tort Statute o Alien Tort Claims Act, se nuclea en torno a las violaciones del Derecho de Gentes, The Law of Nations. 
Ahora bien, el caso que se presenta en estas páginas confirma el cambio de posicionamiento de los tribunales europeos, y demuestra también la fuerte correlación entre los daños medioambientales, las graves violaciones de los Derechos Humanos y la corrupción entre las empresas y los Estados anfitriones de las inversiones ${ }^{25}$. Este nuevo clima, que parece garantizar el acceso a la justicia de las víctimas, se ve reflejado también en el caso ENI, conocido también como Ikebiri por el nombre de la comunidad local afectada, frente a los Tribunales italianos. Cabe mencionar que en el mes de octubre del año 2019 la empresa ENI llegó a un histórico acuerdo con la comunidad de Nigeria de Aggah $^{26}$, para reparar los daños medioambientales causados por la filial nigeriana, NAOC, debido a sus actividades en el territorio de la comunidad que han causado graves impactos medioambientales, sociales y económicos. Durante años, esta comunidad pidió a la empresa filial minimizar los impactos y reparar los daños, sin alcanzar resultado alguno. Por ello, en el año 2018 en un procedimiento frente al Punto Nacional de contacto de Italia, los abogados de la comunidad pidieron a ENI asumir sus responsabilidades por las actividades de las empresas pertenecientes a su grupo $^{27}$. Este procedimiento condujo a la firma del acuerdo entre las partes $^{28}$.

En relación al caso Ikebiri, El 5 de abril de 2010, un oleoducto operado por la Nigerian Agip Oil La compañía (NAOC), bajo el control de ENI S.

25 Sobre este tema Veánse: Wim Huisman y Daniel Sidoli, «Corporations, human rights and the environmental degradation-corruption nexus», Asia Pacific Journal of Environmental Law, Vol. 22, (2019): 66-99. Cabe mencionar que el Profesor Zamora Cabot en su momento estudió esta correlación entre ilícitos contra el medio ambiente, violaciones de derechos humanos y corrupción analizando extensamente el caso Bhopal. Para más información véase: Francisco Javier Zamora Cabot, «Accidentes en masa y forum non conveniens el caso Bhopal», en La responsabilidad internacional. Aspectos de derecho internacional público y derecho internacional privado: XIII Jornadas de la Asociación Española de Profesores de Derecho Internacional y Relaciones Internacionales, ed. por Carlos Jiménez Piernas (Alicante: Asociación Española de Profesores de Derecho Internacional y Relaciones Internacionales, 1989): 533-564.

26 «Nigeria: Una comunidad arranca un acuerdo histórico al gigante petrolero ENI», Federación Internacional por los derechos humanos, acceso el 30 de octubre de 2019, https://www.fidh.org/it/eni-e-la-comunita-nigeriana-di-aggah-hannoraggiunto-uno-storico?fbclid=IwAR37NDB1PxYeqCPP7fORUIdNNtsP0B-yxyh2kC_ UJB8XgAfYcSinN7gv6hs.

27 De esta forma, el abogado italiano Giacomo Cremonesi e rappresentante di FIDH nel procedimento davanti al PCN italiano.

${ }_{28}$ Conciliation procedure in the Specific instance submitted to the Italian NCP by Egbema Voice of Freedom, Chima Williams and Associates (CWA), Advocates for Community Alternatives (ACA) v. ENI s.p.a. 
p. A. que trabaja desde años en Nigeria a través de esta filial, estalló a 250 metros de un arroyo al norte de la comunidad Ikebiri. El derrame afectó los recursos vitales de dicha comunidad esenciales para su supervivencia, contaminando un área importante del territorio. Los representantes legales de la comunidad afectada pidieron a la empresa NAOC que restableciera la situación previa al derrame, eliminado los residuos vertidos, y resarcir a la población. El 18 de abril del año 2010 la filial y la comunidad llegaron a un acuerdo relativo al pago relativo a los relief materials, por el importe de 2.000.000 Naira (unos 6000 euro), pero no sobre la petición de indemnización de los daños.

Para reducir los impactos sociales y sobre el medio ambiente, el representante de la comunidad Ikebiri, junto al abogado Luca Saltalamacchia, presentó una demanda que carecía de precedentes en la práctica judicial italiana contra ENI y su filial Nigerian Agip Oil Company Limited NAOC ${ }^{29}$. Según el abogado italiano, la filial NAOC pertenece al grupo empresarial ENI y se encuentra totalmente bajo el control de la empresa matriz. En otras palabras, sostiene que la empresa matriz toma todas las decisiones relativas a las actividades de la local y, por tanto, también recaen sobre ella obligaciones directas y precisas y debería responsabilizarse por el derrame de petróleo causado por esta última, dado su deber de cuidar toda la estructura corporativa.

Así, en la demanda presentada se evidencia cómo en realidad ENI y NAOC no son dos entidades separadas, aunque esta última se encuentre registrada según la normativa nigeriana. Para demostrar este aspecto, también en este caso se hace referencia a toda la información pública de ENI en la que se muestra NAOC como una empresa sin autonomía alguna en la toma de decisiones operacionales y económicas, que utiliza el mismo logo, la misma página web y donde su consejo de administración está compuesto, en su mayoría, por dirigentes italianos seleccionados o que han trabajado por ENI. Además, se aportan una serie de documentos y declaraciones públicas de ENI en las que se evidencia su papel y labor en Nigeria a través de NAOC, como único ente. En el documento de presentación de la empresa matriz:

Siamo presenti in Nigeria dal 1962 e lavoriamo nel settore Exploration \& Production onshore e offshore. Come sosteneva il nostro primo Presidente Enrico Mattei «il petrolio è loro»:

29 Más información en: «Nigerian Community Sues Italian Oil ENI», Global Campaign to Reclaim Peoples Sovereignty, Dismantle Corporate Power and Stop Impunity, acceso el 10 de enero de 2020, https://www.stopcorporateimpunity.org/nigerian-community-sues-italianoil-giant-eni/. 
per questo condividiamo con gli abitanti del Paese risorse, tecnologie, progetti e li sosteniamo nello sviluppo di nuove attività, in particolare quelle agricole. Nel 2014 la Petroleum Technology Association of Nigeria ha riconosciuto due società Eni come migliori organizzazioni del settore Oil \& Gas nel promuovere lo sviluppo locale; Si legge ancora che dal 18 marzo 2014 NAOC ha attivato un sito web che diffonde in maniera trasparente informazioni su temi di Sostenibilità.[...] In aggiunta a marzo 2015 NAOC ha commissionato a un ente certificatore esterno un audit del proprio Sistema di Gestione degli Oil Spill per verificarne la conformità rispetto alla Legislazione nigeriana, agli standard internazionali e alle linee guida Eni, analizzando 3.900 eventi di Oil spill dal 2007 al 2014.

Según el abogado Saltalamacchia, la Comunidad Ikebiri considera a ENI responsable por ser quien directamente controla y opera en Nigeria a través de NAOC, y también por la falta de mecanismos adecuados de control de las actividades y para la implementación de los principios y normas en términos de due diligence, todo ello unido a la ausencia de instrumentos para prevenir las violaciones de dichas normas y a su negativa a indemnizar a las víctimas y sanear el área afectada.

En relación con la competencia jurisdiccional, el juez italiano tiene capacidad de conocer el asunto respecto de ENI según el art. 3 de la Ley 218/95: «la giurisdizione italiana sussiste quando il convenuto è domiciliato o residente in Italia o vi ha un rappresentante che sia autorizzato a stare in giudizio a norma dell'articolo 77 del codice di procedura civile e negli altri casi in cui è prevista dalla legge», y según el Reglamento Europeo 44/2001 ahora 1215/12 art. 4, sobre los entes domiciliados en el territorio de un Estado miembro. Y asimismo, en relación a NAOC, ya que existiría una correlación entre los hechos y los distintos acusados que justificaría un manejo conjunto de las demandas; es decir, una competencia en base a un foro conexo con una pluralidad de demandados que permitiría atraer a otros responsables frente a los tribunales del domicilio de la matriz, para evitar un riesgo importante de demandas paralelas y decisiones posiblemente contradictorias por órganos judiciales de diferentes Estados, si finalmente estos casos se resuelven por separado.

En la demanda, se solicitó una indemnización de al menos 689,198,400 Naira. También se exigió que los acusados limpiasen el área contaminada y que los niveles de contaminación se redujesen por debajo del umbral prescrito por la ley nigeriana $(50 \mathrm{mg} / \mathrm{kg}$ ). No obstante, en junio de 2019 , las partes llegaron a un acuerdo extrajudicial secreto, por lo que se dio por concluido el procedimiento en Italia contra ENI. 
Pasaré ahora a analizar el caso Kiobel, paradigma de la impunidad que rodea el mundo de las empresas multinacionales ${ }^{30}$, entablado frente a los Tribunales holandeses. Según Amnistía internacional, que apoya al equipo legal de Esther Kiobel, «This case exemplifies how hard it can be for the victims of human rights abuses to hold a powerful multinational corporation to account» ${ }^{31}$.

\section{El caso Kiobel III}

Los tribunales nacionales a menudo desestiman las demandas de las víctimas a partir de una serie de doctrinas que limitan su jurisdicción como, entre otras, la del Forum Non Conveniens ${ }^{32}$, o las conocidas por los tribunales estadounidenses como Political Question Doctrine ${ }^{33}$ o el Acto de Estado ${ }^{34}$, entendiendo en la primera que otro foro, el del lugar donde se verificaron los hechos, es el más adecuado, o conveniens. Pero, a modo de contraste, traemos ahora a colación el caso Kiobel III

En junio de 2017 las viudas de activistas ambientales nigerianos demandaron a Royal Dutch Shell y su filial nigeriana Shell Petroleum and Development Company ante el Tribunal de Distrito de La Haya por graves vio-

30 Vid. Doreen Lustig, «Three Paradigms of Corporate Responsibility in International Law: The Kiobel Moment», Journal of International Criminal Justice, Volume 12, Issue 3,(2014): 593-614, https://academic.oup.com/jicj/article/12/3/593/903020. Liesbeth Enneking, «Transnational Human Rights and Environmental Litigation - A Study of Case Law Relating to Shell in Nigeria.Opent extern», en Feichtner, ed. por Isabel Krajewski, Markus, y Ricarda Roesch, Human Rights in the Extractive Industries - Transparency, Participation, Resistance (Interdisciplinary Studies in Human Rights, 3) (pp. 511-551). Cham: Springer, 2019.

31 «Nigeria/Netherlands: Kiobel witness hearing key chance to hold Shell to account over human rights abuses», Amnisty Internacional, acceso el 30 de octubre de 2019, https:// www.amnesty.org/en/latest/news/2019/10/nigeria-netherlands-kiobel-witness-hearing-keychance-to-hold-shell-to-account-over-human-rights-abuses/.

${ }^{32}$ En relación a los casos Bhopal y Chevron, ver, por ejemplo: José Elías Esteve Moltó, «La estrecha interdependencia entre la criminalidad de las empresas transnacionales y las violaciones al Derecho Internacional de los Derechos Humanos y del Medio Ambiente: Lecciones del caso Bhopal», Revista Electrónica de Estudios Internacionales 32(2016), DOI:1017103/reei.32.01 y, Antoni Pigrau, «The Texaco-Chevron case in Ecuador: Law and justice in the age of globalization», Revista Catalana de Dret Ambiental 5,n. ${ }^{\circ} 1$ (2014).

33 «Boumediene v. Bush and the Political Question Doctrine 99», GEO. L.J. 869, 870 (2011), vid. Anthony J. Colangelo, «De facto Sovereignty: Boumediene and Beyond», GEO. WASH. L. REV. 623, (2009): 667-68.

${ }^{34}$ Kashef v. BNP Paribas S.A., No. 18-1304 (2d Cir. 2019), US Court of Appeals for the Second Circuit. 
laciones contra los Derechos Humanos que tuvieron lugar en la región de Ogoniland en el Delta del Níger a mediados de la década de 1990, cuando los residentes protestaban contra la degradación ambiental causada por las actividades de esas empresas relacionadas con la extracción y producción de petróleo en la zona. Ken-Saro Wiwa, una figura prominente entre los defensores africanos de los Derechos Humanos y otros ocho activistas fueron sentenciados a la pena capital y ejecutados en la horca, tras una parodia de proceso a cargo del corrupto régimen militar que entonces regía el país y a instancias, se denunciaba, de las citadas empresas. Los familiares de las víctimas iniciaron entonces dos diferentes demandas en los Estados Unidos (EE. UU.), las mismas que, junto a una nutrida doctrina, hemos tenido la oportunidad de comentar en trabajos previos ${ }^{35}$.

Estas demandas se fundamentaron, entre otras normas, en el Alien Tort Claims Act (ATCA) ${ }^{36}$, contra la petrolera Shell por haber instigado al gobierno nigeriano a cometer dichos abusos. En junio de 2009, Shell llegó a un acuerdo extrajudicial con respecto de la demanda presentada por el hijo de $\mathrm{Wiwa}^{37}$. Mientras que el segundo litigio, presentado por algunas viudas, entre ellas Esther Kiobel, continuó hasta abril de 2013,

35 Vid., entre otros, Maria Chiara Marullo, y Francisco Javier Zamora Cabot «Transnational Human Rights litigation. Kiobel's Touch and concern: a test under construction », Papeles el Tiempo de los Derechos y la bibliografia allí citada. El documento se puede consultar en: https://papers.ssrn.com/sol3/papers.cfm?abstract_id=2765068. Acceso el 16 de octubre de 2019.

También, Anna Grear, and Weston, Burns H, «The Betrayal of Human Rights and the Urgency of Universal Corporate Accountability: Reflections on a Post-Kiobel Lawscape», 15 Human Rights Law Review, (2015): 21-44 .También, Liesbeth Enneking, «Multinational Corporations, Human Rights Violations and a 1789 US Statute - A Brief Exploration of the Case of Kiobel v. Shell», Nederlands Internationaal Privaatrecht, (2012), 396-400.

36 ATS, Judiciary Act of 1789, ch. 20, §9(b), 1 Stat. 73, 77 (1789), codified in 28 U.S.C. Sec. 1350; esta norma ha sido utilizada para prevenir y reparar esas conductas perpetradas tanto por individuos como por empresas, desde la perspectiva civil. En otras palabras, en las últimas décadas a través de este ATCA se ha otorgado una respuesta reparadora, indemnizaciones, a las víctimas, en un País, Estados Unidos (EE.UU.), que no necesita estar directamente relacionado con la conducta ilícita que ha provocado un daño o prejudicio como grave violación a los Derechos Humanos. Sin embargo, este sistema se ha visto menoscabado por decisiones jurisprudenciales que han vaciado casi de contenido el ATCA y con pocas posibilidades de ser aplicado respeto de conductas que se verifican fuera o que no tengan una fuerte conexión con el territorio de los Estados Unidos. Vid. Entre otros, Carmen Márquez Carrasco, «Las relaciones entre el Derecho internacional y la práctica interna en el ámbito de los Derechos Humanos y la responsabilidad de las empresas», Anuario español de derecho internacional 34, (2018): 707-728 y Marta Requejo Isidro, «Responsabilidad civil y Derechos Humanos en EEUU: ¿el fin del ATS?», Indret: Revista para el Análisis del Derecho, n. $^{\circ} 3,(2011)$.

37 Wiwa v. Royal Dutch Petroleum Co, US District Court for the Southern District of New York, 99-7223 
cuando el Tribunal Supremo de los Estados Unidos, sobre la base de la presunción contra la extraterritorialidad, afirmó en Kiobel $I I^{38}$ que el ATCA no puede ser aplicado respecto de conductas que se verifican fuera o que no tengan una fuerte conexión con el territorio de los EE.UU., ya que «el ATS no se extiende contra empresas extranjeras cuando toda la conducta relevante aconteció fuera de los Estados Unidos», confirmando por tanto una sentencia de 2010 del Tribunal de Apelaciones del Segundo Circuito Federal, Kiobel ${ }^{39}$, en la que el caso había sido desestimado por falta de jurisdicción. Este posicionamiento se ha visto respaldado por otras decisiones, como en el caso Daimler ${ }^{40}$ en el que este Tribunal va, en la práctica, más allá, al introducir en el ámbito de la llamada Jurisdicción Personal General, General Personal Jurisdiction, y respecto de las empresas, el requisito de que las extranjeras solo puedan ser demandadas donde se sientan essentially at home lo que, en realidad, abona limitar la competencia sobre ellas a los tribunales de países en los que están registradas o tengan su administración principal. Por su parte, en la decisión en el caso Jesner ${ }^{41}$ da un paso más, e importante, en este proceso de restricción del acceso a los tribunales de los Estados Unidos en litigios sobre violaciones de los derechos humanos, haciéndolos imposibles respecto de las empresas extranjeras y, probablemente, preparando el terreno para que suceda lo propio con las domésticas.

Ahora bien, descartada la vía de reparación en los Estados Unidos en el caso descrito, la nueva demanda presentada por Esther Kiobel y sus compañeras en Holanda y admitida ya a trámite por los tribunales neerlandeses, podría suponer, de fructificar, un nuevo hito en la naciente jurisprudencia que está abriéndose camino en varios países europeos, en el sentido de luchar contra la impunidad de gravísimas conductas contrarias a los derechos humanos, manteniendo así el imperio de la ley y su obligado corolario, el

${ }^{38}$ Kiobel v. Royal Dutch Petroleum Co, Supreme Court of the United States 569 U.S No. 10-1491.

${ }^{39}$ Kiobel v. Royal Dutch Petroleum, Appeal judgment, 06-4800-cv, o6-4876-cv, 17-IX2010.

${ }^{40}$ Daimler AG v. Bauman et al, Supreme Court of the United States No. 11-965. Sobre este tema: Francisco Javier Zamora Cabot, «Decision of the Supreme Court of the United States in the Daimler AG v. Bauman Et Al Case: Closing the Golden Door», Papeles el Tiempo de los Derechos 2 (2014), https://papers.ssrn.com/sol3/papers.cfm?abstract_ id $=2791635$.

${ }^{41}$ Justice Kennedy «Jesner v. Arab Bank, PLC», Scotus Blog, 24 de Abril, http://www. scotusblog.com/casefiles/cases/jesner-v-arab-bank-plc/

Sobre este tema: Francisco Javier Zamora Cabot y Maria Chiara Marullo, «Never send to know..., Comentario crítico a la sentencia Jesner V. Arab Bank, del Tribunal Supremo de los Estados Unidos», Papeles el Tiempo de los Derechos 32 (2019), http://repositori.uji.es/xmlui/ handle/10234/176940. Acceso el 16 de octubre de 2019. 
acceso de las víctimas a la justicia ${ }^{42}$. Tratándose de la jurisdicción, la acción se presenta contra:

-Royal Dutch Shell Plc of London, United Kingdom, with its registered office in the Hague, -Shell Petroleum n.v. of the Hague, - the shell transport and trading company limited of London, - the Shell Petroleum development company of Nigeria ltd of Port Harcourt, Rivers State, Federal Republic of Nigeria.

En relación con la empresa matríz, domiciliada en Holanda y la domiciliada en el Reino Unido, se señala por el Tribunal holandés que «It is not in dispute that the Dutch court has international jurisdiction to take cognizance of the claims against the parties RDS and SPNV, established in the Netherlands. The court has international jurisdiction to take cognizance of the claims against STTC, established in London». Y, respecto de la filial, este Tribunal reconoce su jurisdicción, puesto que el primer demandado está domiciliado en los Países Bajos, y ello en base al Artículo $7 \mathrm{CCP}^{43}$, dado que existiría una correlación entre los hechos y los distintos acusados que justificaría un manejo conjunto de las demandas; en otras palabras, un foro conexo con una pluralidad de demandados que permitiría atraer a otros responsables frente a los tribunales del domicilio de la matriz:

SPDC, established in Nigeria, if the requirements of Article 8 paragraph 1 Brussels I bis Regulation10 and of Article 7 Code of Civil Procedure (CCP), respectively, have been met. [...] Under Article 7 $C C P$, jurisdiction as regards SPDC can be assumed if the correlation between the claims against the various defendants is such that reasons of efficiency justify a joint handling of the claims.

42 Matthias Weller, Alexia Pato, «Local parents as 'anchor defendants' in European courts for claims against their foreign subsidiaries in human rights and environmental damages litigation: recent case law and legislative trends», Uniform. Law Review, 23 (2018): 397-417.

43 Article 7 Jurisdiction over counter actions, joinders and interventions

-1 . If legal proceedings are to be initiated by a writ of summons and a Dutch court has jurisdiction with respect to one of the defendants, then it has jurisdiction as well with respect to the other defendants who are called to the same proceedings, provided that the rights of action against the different defendants are connected with each other in such a way that a joint consideration is justified for reasons of efficiency.

-2 . If legal proceedings are to be initiated by a writ of summons and a Dutch court has jurisdiction over the legal claim, then it has jurisdiction as well over a counter action (counterclaim) and over a right of action against a third party who is called to the proceedings by a defendant as being the ultimate liable person, and over a right of action of a third party who has appeared in court for a consolidation of actions ('joinder') or an intervention, unless there is not enough connection between these other actions and the original action. 
En otro orden, el Tribunal afirma que los demandantes actuaron de forma concertada respecto de las violaciones alegadas y las acciones contra ellos se basan en los mismos hechos, circunstancias y bases legales, por lo que existe un riesgo importante de que se tomen decisiones contradictorias por órganos judiciales de diferentes Estados, si finalmente estos casos se dirimen por separado:

The heart of the accusation of claimants is that SPNV, STTC and $S P D C$ acted in concert in the fundamental rights violations alleged by claimants. The claims against the three defendants are based on the same facts, circumstances and legal bases. The claims against SPNV, STTC and SDPV therefore pertain to the same situation, both factually and at law. This is especially true for the claims against the two parent companies SPNV and STTC, which both factually and legally cannot be viewed in isolation from the claims against the operating company $S P D C$. The court is of the opinion, like claimants, that if these related cases were to be prosecuted separately, there is a risk that contradictory decisions will be made.

De esta sentencia destacamos además otros dos elementos que consideramos del mayor relieve; el primero, la calificación de los hechos por parte del Tribunal como violaciones de Derechos Humanos y no sobre meros ilícitos civiles internacionales ${ }^{44}$. Y el segundo, que haya ordenado también el uso limitado de los documentos obtenidos en los procedimientos frente a los tribunales de los EE. UU ${ }^{45}$.

Sobre este último particular, tras la sentencia de 2013 en los EE.UU. Esther Kiobel, en preparación de la demanda en Holanda, presentó en 2016 ante la jurisdicción federal del país transatlántico una petición relativa a la conocida 28 U.S.C. Section 1782 (a), sobre obtención de pruebas en el extranjero, pidiendo todas las trascripciones y las pruebas obtenidas en los procedimientos Wiwa y Kiobel I y II, así como todos los documentos producidos por los demandados. En sus términos, la Section 1782 (a) preceptúa que:

${ }^{44}$ Más información en: Geert Van Calstet, 17 de mayo de 2019(8:08), Kiobel v Shell in The Netherlands. Court confirms jurisdiction anchored unto mother holding and qualifies the suit as one in human rights: not tort. Also orders limited use of documents obtained in US discovery and limited continuation of the trial, Gavc Law-Geert Van Calster, 10 de enero de 2020, https://gavclaw.com/2019/05/17/kiobel-v-shell-in-the-netherlands-court-confirmsjurisdiction-anchored-unto-mother-holding-and-qualifies-the-suit-as-one-in-human-rightsnot-tort-also-orders-limited-use-of-documents-obtained-in-us/.

${ }^{45} \mathrm{La}$ versión en inglés de la demanda se puede consultar en: https://uitspraken. rechtspraak.nl/inziendocument?id=ECLI:NL:RBDHA:2019:6670. Acceso el 10 de enero de 2020 . 
Provide [s] federal-court assistance in gathering evidence for use in foreign tribunals. [...] The district court of the district in which a person resides or is found may order him to give his testimony or statement or to produce a document or other thing for use in a proceeding in a foreign or international tribunal, including criminal investigations conducted before formal accusation. The order may be made . . . upon the application of any interested person and may direct that the testimony or statement be given, or the document or other thing be produced, before a person appointed by the court.[...] 28 U.S.C. $\S$ 1782(a). Section 1782 states that a court «may order» such discovery; so even if a court has jurisdiction under the statute to grant a petition, the decision to grant it is discretionary ${ }^{46}$.

El Juzgado de Distrito concluyó que tenía jurisdicción para considerar la petición de Kiobel, rechazando los argumentos de Shell y finalmente admitió la petición ${ }^{47}$. En la apelación, los abogados de la empresa cuestionan tanto la jurisdicción como la concesión discrecional de la petición. En cuanto a la jurisdicción, sostienen, entre otras cosas, que los documentos pertenecen a Shell y que la empresa no reside ni se encuentra en el Distrito Sur de Nueva York (el del Juzgado). En cuanto a la concesión discrecional de la petición, argumentan que los materiales producidos por Shell estaban cubiertos por la confidencialidad. Finalmente, el Tribunal de Apelación estimó los argumentos del demandado por lo que considera abuso de discrecionalidad del Juzgado de Distrito al otorgar la petición y el intento de Kiobel de eludir las normas sobre la confidencialidad de los documentos ${ }^{48}$. El

46 United States Court of Appeals for the Second Circuit August Term 2017 No. 17424 cv, Esther Kiobel, by her attorney in fact Channa Samkalden, Petitioner Appellee, v. Cravath, Swaine \& Moore llp Respondent Appellant. 1, en: http://www.ca2.uscourts.gov/decisions/ isysquery/b80ffeca-f146-49ce-be16-d6c2219bcb3d/2/doc/17-424_opn.pdf\#xml=http://www . ca2 .uscourts.gov/decisions/isysquery/b80ffeca-f146-49ce-be16-d6c2219bcb3d/2/hilite/. Acceso el 10 de enero de 2020.

47 Discovery. Section 1782. District court grants petition seeking documents from law firm to be used in an anticipated civil action by Petitioner against the law firm's client in the Netherlands pursuant to 28 U.S.C. $\$ 1782$. En: http://bakerxchange.com/

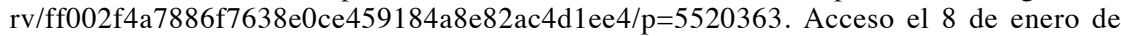
2020 .

48 «Discovery Section 1782. Second Circuit reverses a district court order granting a Section 1782 discovery petition where Petitioner sought to subpoena documents belonging to the client of a US law firm for use in a foreign proceeding», Baker McKenzie, International litigation \& Arbitration, acceso el 10de enero de 2020

http://bakerxchange.com/rv/ff00411acbc3c2f7df0fbb9b02449eb56107e899/p=3097517. Más información en: https://www.mayerbrown.com/en/perspectives-events/publications/2018/07/second-circuit-limits-use-of-section-1782-discover. Acceso el 8 de enero de 2020. 
28 de noviembre de 2018, Esther Kiobel presenta una Petition for a write of certiorari $^{49}$, denegada por el Tribunal Supremo el 7 de enero de $2019^{50}$.

Ahora bien, el Tribunal holandés en la sentencia de mayo 2019, ordena un uso limitado de los documentos producidos por las partes en procedimientos previos, así como de las comunicaciones entre las empresas y otros documentos. Cabe por tanto hacer una distinción entre las categorías de pruebas y documentos. En la sentencia se establece que:

4.30. The claims in the procedural issue relate to two categories of documents from the discovery phase in the American proceedings: the intended trial exhibits and intended trial depositions (claim i) and ii)) and the CDST documents (claim iii)). At the hearing, claimants extended their claim to the unredacted version of the minutes of the meeting of the CMD of 7 November 1995, of which defendants submitted a redacted version to the proceedings.

En relación a the intended trial exhibits and intended trial depositions (claim i) and ii)), estos elementos de la reclamación pertenecen a las pruebas documentales en los procedimientos frente a los tribunales estadounidenses. Estos documentos fueron producidos por las compañías pertenecientes al Grupo Shell, que eran parte en los procedimientos estadounidenses, y tuvieron que ser devueltos o destruidos dentro de los 30 días posteriores a la finalización de los mismos en virtud del acuerdo de confidencialidad entre los demandantes y las empresas Shell. No obstante, los demandantes solicitan ahora tener acceso a una parte importante de ellos debido a que el nuevo caso, frente al Tribunal holandés, se fundamenta en los mismos hechos y las mismas violaciones. Este Tribunal afirma que las pruebas documentales se emitieron previamente en los procedimientos estadounidenses en virtud del acuerdo de confidencialidad, y se refieren a acciones judiciales diferentes a la que lo ocupan en el caso. Además, las pruebas se definen de manera demasiado amplia y no cumplen con el requisito de identificar documentos específicos y tampoco se puede suponer que se ha cumplido el requisito de interés legítimo, según la Sección 843a subsección $1 \mathrm{CC}$, de la ley procesal holandesa, por lo que rechaza la solicitud.

Por su parte, en relación con los documentos bajo el claim o pretensión iii), la sede judicial holandesa afirma que:

49 Más información en: https://earthrights.org/wp-content/uploads/Kiobel-cert-petition. pdf. Acceso el 10 de enero de 2020.

${ }^{50}$ Mas información en: Esther Kiobel, By and Through Her Attorney-In-Fact, Channa Samkalden, Petitioner v. Cravath, Swaine \& Moore LLP, Supreme Court of the United States, acceso el 10 de enero de 2020, https://www.supremecourt.gov/search.aspx ?filename=/ docket/docketfiles/html/public/18-706.html. 
The CDST documents claimed under iii) are the documents pertaining to the tribunal, which in the privilege log in the discovery of the American proceedings have been described as Communications from counsel regarding proceedings before the Ogoni Civil Disturbances Tribunal. These are (i) the trial reports, the reports of the attorneys of the office of [attorney 1], who had been hired by SPDC to monitor the proceedings before the tribunal [...] (ii) documents which reported on the content of the trial reports within SPDC or wider within the Shell Group. [...] ii) CDST documents designated in 3.3 under iii). The dispute is limited to these documents, which defendants have not issued.

En términos generales, los demandados afirman que estos documentos contienen información comercial confidencial, por lo que son los demandantes quienes tienen que demostrar que en un caso concreto existe un motivo convincente para aceptar la solicitud con respecto a esa información confidencial. Pero, según se estima por el Tribunal, es en realidad la parte que se opone a la solicitud la que debe argumentar convincentemente sobre la existencia de su interés en la confidencialidad y, al no haberse cumplido en el caso con tal requisito:

This means that defendants must issue the CDST documents stated in claim iii) to claimants, within the claimed term of 21 days. The claimed penalty is awarded as being insufficiently disputed. The internal nature of the documents compels the court to attach the condition that claimants only use these documents for these proceedings, pursuant to Section 28 CCP. Claimants are also prohibited from issuing the documents to third parties or disclose anything about the content of these documents to third parties.

Concluyendo, en relación con el acta de la reunión del 7 de noviembre de 1995 que:

Finally, at the hearing claimants have extended their claim in the procedural issue to include the non-redacted minutes of the meeting of the CMD of 7 November 1995, of which defendants submitted a redacted version. The court understands that claimants also wish to receive an excerpt of the redacted passages, in so far as they are related to the tribunal and/or the Ogoni issue.

\section{Conclusiones}

La protección de los Derechos Humanos y del medio ambiente en la región del Delta del Niger, afectados por las actividades empresariales extractivas desarrolladas principalmente por dos grandes empresas que operan en 
la región a través de sus filiales, Shell y ENI, sigue siendo un tema de vital importancia para la comunidad local, nacional e internacional. En ámbito internacional son muchos los estándares y las normas aceptadas, además de las buenas prácticas relativas al desempeño de las actividades de extracción de petróleo. Por ejemplo, la International Petroleum Industry Environmental Conservation Association ha desarrollado una guía para evaluar el impacto social y ambiental relativo al derrame. Tanto Shell como ENI son miembros de esta asociación por lo que deberían, de forma voluntaria, cumplir con dichos estándares y establecer acciones preventivas, para evitar dichos impactos y poner en marcha mecanismos para remediar, en los casos en los que se verifican los derrames.

Al mismo tiempo, los Estados, principalmente Nigeria y los países de origen de las empresas, deberían dar efectividad a dichos estándares y velar por su cumplimiento, garantizando en cualquier caso, responsabilizar a las corporaciones cuando se pueda demostrar que han fallado en la activación de medidas preventivas y después, por no remediar adecuadamente por los daños provocados. Con ello, deberían garantizar siempre el acceso a la justicia de las víctimas.

Somos conscientes de que para lograr un cambio real en materia de acceso a la justicia y de prevención y reparación de daños, son necesarias acciones legales, estatales e internacionales vinculantes y que, por ejemplo, permitan sancionar de forma más contundente, también penalmente en su caso, a las empresas multinacionales por las graves violaciones perpetradas fuera de sus países de origen. Sería por tanto oportuno seguir avanzando en la creación de un instrumento internacional vinculante, el Binding Treaty on Business and Human Rights ${ }^{51}$, y también en la vía de nuevas propuestas legislativas como, entre otras, sendas iniciativas legislativas suizas relativas a las responsabilidades de las empresas multinacionales por graves violaciones de los Derechos Humanos y por daños medioambientales ${ }^{52}$

51 Sobre este tema: Adoración Guamán, «Derechos humanos y empresas transnacionales: las debilidades del tercer pilar derivadas de las normas de promoción de inversiones. El caso Chevron como paradigma de la necesidad del Binding Treaty», Cuadernos Electrónicos de Filosofía del Derecho 39, (2019), https://ojs.uv.es/index.php/ CEFD/article/view/13988. También, resultan de gran interés los trabajos del profesor Larry Catá Backer relativos a la propuesta de Tratado internacional sobre empresas y Derechos Humanos, que se pueden consultar en su blog: http://lcbackerblog.blogspot.com/. Acceso el 10 de enero de 2020.

52 'Conseil National Session de Printemps 2015 Neuvième Séance 11.03.15 15h00 14.3671' (2015): Mise en oeuvre du rapport de droit comparé du Conseil fédéral sur la responsabilité des entreprises en matière de droits humains et d'environnement, acceso el 7 de enero de 2020, https://www.parlament.ch/fr/ratsbetrieb/amtliches-bulletin/amtliches- 
Al mismo tiempo, opinamos que hay que seguir utilizando los mecanismos legales y procesales existentes para, por lo menos, indemnizar a las víctimas y reparar los daños. Ponemos de relieve que en el ámbito europeo y respecto de litigios civiles, si solo fuera por los ejemplos que hemos destacado, estaríamos ante un momento de arraigo y consolidación, que consideramos merece un seguimiento estrecho y, si nos cabe decirlo, esperanzado. Responsabilizar a las empresas por delitos graves que afectan a la humanidad en su conjunto no solo contribuiría a poner fin a su impunidad, sino también a reducir el desequilibrio de poderes entre los países de los que proceden y aquellos donde sus empresas llevan a cabo sus actividades, en numerosas ocasiones con graves y del todo inasumibles efectos respecto del medio ambiente y los Derechos Humanos ${ }^{53}$.

\section{Sobre la autora}

Maria Chiara Marullo es Profesora Ayudante Doctor de Derecho Internacional Privado, Facultad de Ciencias Jurídicas y Económicas, Departamento de Derecho Privado de la Universitat Jaume I. Acreditada a Profesora Contratada Doctora. Miembro de la REDH-EXATA, redhexata.com. Doctora cum laude por la Universitat Jaume I (2014) y Premio Jaime Brunet a la mejor tesis doctoral a la promoción de los Derechos Humanos, Universidad Pública de Navarra (2016). Investigadora postdoctoral en la Facultad de Ciencias Jurídicas y Económicas, Departamento de Finanzas y Contabilidad. Grupos de investigación: «Sostenibilidad de las Organizaciones y Gestión de la Responsabilidad Social - Mercados Financieros» y «Derechos Humanos y Derechos Fundamentales». Investigadora en el proyecto «SMART Sustainable Market Actors for Responsible Trade» (2016-2020); colaboradora del proyecto «Constitución climática global: gobernanza y Derecho en un contexto complejo» (2017-2019) e investigadora en el Proyecto Europeo «Human Rights in Business» (2014-2016).

bulletin-die-verhandlungen?SubjectId=35082. Sobre este tema véanse también: Dalia Palombo, Business and Human Rights: The Obligations of the European Home State (Oxford: Hart Publishing, 2020).

${ }^{53}$ Una interesante reflexión en: «Is criminal responsibility of companies the next focus for international justice?» https://www.justiceinfo.net/en/justiceinfo-comment-and-debate/ opinion/41943-is-criminal-responsibility-of-companies-the-next-focus-for-internationaljustice.html. Acceso el 8 de enero de 2020, 


\section{About the autor}

Maria Chiara Marullo is an Assistant Professor of Private International Law, Law Faculty, Department of Private Law, Universitat Jaume I. Member of the network: REDH-EXATA, redhexata.com. Doctor cum laude from the Universitat Jaume I (2014) and Jaime Brunet Award for the best doctoral thesis for the promotion of Human Rights, Public University of Navarra (2016). Postdoctoral Researcher, Department of Finance and Accounting, Universitat Jaume I. Research group: «Sustainability of Organizations and Management of Social Responsibility - Financial Markets» and «Human Rights and Fundamental Rights». Researcher in the «SMART Sustainable Market Actors for Responsible Trade» European Project (20162020); collaborator in the project: «Global climate constitution: governance and law in a complex context» (2017-2019), and researcher in the European Project «Human Rights in Business» (2014-2016). 


\section{Derechos de autor}

Los derechos de autor (para la distribución, comunicación pública, reproducción e inclusión en bases de datos de indexación y repositorios institucionales) de esta publicación (Cuadernos Europeos de Deusto, CED) pertenecen a la editorial Universidad de Deusto. El acceso al contenido digital de cualquier número de Cuadernos Europeos de Deusto es gratuito inmediatamente después de su publicación. Los trabajos podrán leerse, descargarse, copiar y difundir en cualquier medio sin fines comerciales y según lo previsto por la ley; sin la previa autorización de la Editorial (Universidad de Deusto) o el autor. Así mismo, los trabajos editados en CED pueden ser publicados con posterioridad en otros medios o revistas, siempre que el autor indique con claridad y en la primera nota a pie de página que el trabajo se publicó por primera vez en CED, con indicación del número, año, páginas y DOI (si procede). Cualquier otro uso de su contenido en cualquier medio o formato, ahora conocido o desarrollado en el futuro, requiere el permiso previo por escrito del titular de los derechos de autor.

\section{Copyright}

Copyright (for distribution, public communication, reproduction and inclusion in indexation databases and institutional repositories) of this publication (Cuadernos Europeos de Deusto, CED) belongs to the publisher University of Deusto. Access to the digital content of any Issue of Cuadernos Europeos de Deusto is free upon its publication. The content can be read, downloaded, copied, and distributed freely in any medium only for non-commercial purposes and in accordance with any applicable copyright legislation, without prior permission from the copyright holder (University of Deusto) or the author. Thus, the content of CED can be subsequently published in other media or journals, as long as the author clearly indicates in the first footnote that the work was published in CED for the first time, indicating the Issue number, year, pages, and DOI (if applicable). Any other use of its content in any medium or format, now known or developed in the future, requires prior written permission of the copyright holder. 